\title{
Neuralgia del trigémino
}

\author{
G.R. Boto
}

Servicio de Neurocirugía. Hospital Clínico "San Carlos”. Facultad de Medicina. Universidad Complutense. Madrid.

\section{Resumen}

La neuralgia del trigémino continúa siendo una enfermedad desconocida para muchos médicos generales y lo que resulta aun peor, mal manejada por muchos de los especialistas encargados de la misma.

En este trabajo se resumen las principales características anatomo-clínicas, fisiopatológicas y terapéuticas de esta entidad.

PALABRAS CLAVE: Neuralgia del trigémino. Dolor facial.

\section{Trigeminal neuralgia}

\section{Summary}

Trigeminal neuralgia continues to be an illness poorly known for many general practitioners and what is even worse, badly handled by many of the specialists in charge of cephalic neuralgias.

In this paper I resume the main knowledge about trigeminal neuralgia reviewing the clinical, physiopathological, and therapeutical aspects of this condition.

KEY WORDS: Trigeminal neuralgia. Facial pain.

\section{Introducción}

La neuralgia del trigémino o tic douloureux es un dolor facial paroxístico, lancinante, que se describe como una descarga eléctrica, de segundos de duración (raramente llega al minuto), a menudo desencadenado por un estímulo sensorial en zonas de la cara específicas (las llamadas zonas trigger o gatillo) y distribuido por el territorio de inervación de una o más ramas del nervio trigémino. Así, el dolor aparece al comer, al lavarse los dientes, al tocarse la cara, con el aire frío,... Característicamente, la descarga dolorosa no

Recibido: 1-02-10. Aceptado: 1-03-10 es nocturna ya que durante el sueño no se estimulan estas zonas gatillo. El dolor se repite a intervalos irregulares, existiendo por tanto períodos libres de dolor de semanas o meses de duración, que son característicos, de tal manera que la ausencia de estos intervalos sin dolor pone en duda el diagnóstico de neuralgia del trigémino. Típicamente, la enfermedad tiende a la remisión espontánea con el tiempo, al menos inicialmente.

\section{Aspectos clínicos y diagnósticos}

Característicamente es una enfermedad que aparece por encima de los 50 años (promedio: 63 años). Para algunos autores es más frecuente en varones $(1,2: 1)^{46}$, mientras que para otros lo es en mujeres ${ }^{21}$. La incidencia anual se estima en 4 por 100.000 habitantes. Excepcionalmente es familiar.

Lo más frecuente es que el dolor afecte a la hemicara derecha ( $60 \%$ de los casos); en el $39 \%$ de los casos se presenta en el lado izquierdo; es bilateral en el $1 \%{ }^{46}$. En los casos de bilateralidad, el dolor suele aparecer de manera alternante. La esclerosis múltiple es el factor predisponente más importante para presentar una neuralgia bilateral, de tal manera que aproximadamente el $18 \%$ de los pacientes con neuralgia del trigémino bilateral padecen esclerosis múltiple.

La $2^{\mathrm{a}}$ y la $3^{\mathrm{a}}$ ramas del trigémino, de manera conjunta, son las más frecuentemente involucradas $(42 \%$ de los casos); luego la $2^{\mathrm{a}}$ rama aisladamente (20\%); en el $17 \%$ de los casos se afecta la $3^{\mathrm{a}}$ rama; en el $14 \%$ la $1^{\mathrm{a}}$ junto a la $2^{\mathrm{a}}$; la afectación de las tres ramas simultáneamente ocurre en el $5 \%$; la distribución más rara corresponde a la $1^{\mathrm{a}}$ rama $(2 \%$ de los casos) ${ }^{46}$.

Rara vez la neuralgia trigeminal se manifiesta como estatus trigeminal, que consiste en una rápida sucesión de espasmos, como tics, desencadenados aparentemente por cualquier estímulo; el tratamiento con fenitoína intravenosa puede ser útil en estos casos.

Por otro lado, la neuralgia del trigémino puede ser primaria (denominada idiopática o esencial, por otros) o secundaria (también conocida como sintomática). 
Es primaria cuando no se descubre una causa que explique el cuadro; éstas, las primarias, son las más frecuentes.

Las secundarias, por definición, son aquéllas en las que se descubre una causa subyacente. Su clínica dominante son las parestesias y las disestesias, pasando el dolor a formar parte del cuadro de manera secundaria. Además, usualmente se descubren signos deficitarios en la exploración neurológica. Las secundarias dan pues, con frecuencia, un cuadro de neuralgia atípica pero a veces son indistinguibles de la esencial al menos al comienzo ${ }^{35}$. Entre las causas de neuralgia secundaria se encuentran las lesiones del ángulo pontocerebeloso, cuadros que afectan al tronco cerebral, patología diversa del cavum de Meckel, tumores de la fosa media, metástasis de la base craneal, adenomas pituitarios y otras más, siendo la más frecuente de todas ellas, el neurinoma del acústico. Además, los tumores de la fosa posterior son las lesiones que con más probabilidad causan neuralgias que parecen verdaderas o típicas. Otra lesión que puede dar una neuralgia del trigémino de características similares a la esencial es la esclerosis múltiple, de tal forma que aproximadamente el $2 \%$ de los pacientes con esclerosis múltiple tienen una neuralgia de este tipo.

Mención especial merece la asociación entre tumor cerebral y neuralgia del trigémino. La probabilidad de tener un tumor cerebral en el seno de una neuralgia del trigémino es menor del $0,8 \%$. Cabe mencionar que el dolor facial puede ser contralateral al tumor, probablemente debido al desplazamiento que induce la masa en el tronco cerebral. Cuando la neuralgia del trigémino es causada por un tumor cerebral suele tener características atípicas, normalmente el dolor es constante, se descubren en la exploración neurológica signos deficitarios, principalmente pérdida de sensibilidad (aunque en algunos enfermos la exploración puede ser rigurosamente normal en un principio) y el cuadro doloroso aparece en un sujeto relativamente joven (recordemos que la neuralgia del trigémino típica afecta generalmente a personas mayores de 50 años). Además, los sujetos con neuralgia típica del trigémino característicamente responden a carbamazepina en un principio, lo cual no ocurre si el dolor facial es atípico ${ }^{6}$.

La exploración de un paciente con neuralgia del trigémino debe incluir la sensibilidad facial, la musculatura extrínseca ocular, la función de los maseteros (la masticación) y la de los pterigoideos (con la boca abierta, el mentón se desvía al lado enfermo en caso de paresia).

$\mathrm{Si}$ en la exploración se descubre cualquier déficit neurológico en un paciente no operado previamente, se deberá sospechar una causa estructural como origen del dolor neurálgico (tumor, esclerosis múltiple...). Así, ante cualquier neuralgia del trigémino con clínica atípica, se debe realizar una RM craneal. No obstante, dado que como hemos explicado antes es posible que exista una neuralgia del trigémino típica asociada a una lesión estructural cerebral, la realización de RM craneal resulta mandatoria ante cualquier neuralgia del trigémino.

En el diagnóstico diferencial de la neuralgia del trigémino se debe incluir: el herpes zoster (el dolor es continuo, no paroxístico, las típicas vesículas y costras aparecen tras el dolor y éste se distribuye con más frecuencia por el territorio de la primera rama trigeminal (en esta distribución, recordad que la neuralgia del trigémino es muy rara); no obstante, en los casos de herpes zoster donde no aparecen vesículas, el diagnóstico diferencial puede resultar difícil); la enfermedad dental; la patología orbitaria; la arteritis de la temporal (típicamente aparece hipersensibilidad sobre la arteria temporal superficial) y los tumores intracraneales.

\section{Aspectos anatomofisiológicos}

Los receptores sensoriales del nervio trigémino recogen los estímulos aferentes de la piel, mucosa, husos musculares y articulaciones. Estos receptores son del mismo tipo que los de los nervios espinales. Entre ellos están los nociceptores que son estructuras especializadas en la percepción de estímulos nociceptivos, formados por axones del tipo A finamente mielinizados con un umbral elevado de activación mecánica ${ }^{47}$.

El sistema trigeminal inerva además, dos estructuras con receptores sensoriales especializados: la córnea y las encías $^{18}$. La inervación de la córnea depende de fibras aferentes A finamente mielinizadas (delta), y la de las encías de fibras aferentes A finamente mielinizadas (delta) y de fibras amielínicas (fibras C). Ambas estructuras transmiten casi exclusivamente información nociceptiva.

Las fibras aferentes de los receptores sensitivos se agrupan en tres nervios periféricos, que son el oftálmico (V1), el maxilar (V2) y el mandibular (V3).

Los cuerpos neuronales de las fibras aferentes primarias del trigémino están contenidos en el ganglio semilunar o de Gasser, el cual, se encuentra situado en una excavación del ápex del peñasco rodeado de las cubiertas meníngeas, formando el llamado cavum de Meckel. Detrás del ganglio se encuentra la cisterna trigeminal, la cual contiene líquido cefalorraquídeo; dicha cisterna comunica con las cisternas de la fosa posterior a través del denominado porus trigemini. El ganglio de Gasser está organizado somatotópicamente en sentido medial a lateral ${ }^{18}$, de tal manera que las neuronas de la rama oftálmica se sitúan anteromedialmente, las de la rama mandibular posterolateralmente y las de la rama maxilar en medio de las otras dos. Si bien las ramas oftálmica y maxilar están bien separadas, entre la maxilar y mandibular existe cierto grado de solapamiento ${ }^{47}$.

La raíz del trigémino transcurre desde el ganglio de Gasser hasta la protuberancia; dicha raíz está formada por 
una portio major o sensorial, una portio minor o motora y una portio intermedia o accesoria. En la portio major se mantiene la misma organización somatotópica que en el ganglio de Gasser. No obstante, existe una considerable variación en esta distribución y más si tenemos en cuenta la distorsión progresiva de la raíz por rotación a medida que se aproxima al tronco cerebral, de tal manera que cerca de la protuberancia las fibras de V1 quedan superiores y las de V3 inferiores ${ }^{18}$.

La portio major, o rama sensorial, está compuesta por unas 125.000 fibras, de las cuales sólo el 50\% son amielínicas, mientras que en las raíces dorsales espinales el $80 \%$ de las fibras son amielínicas. Se desconoce el significado de esta diferencia. A medida que recorremos la portio major en dirección central, observamos que existe una zona de transición entre la porción de raíz cubierta por mielina periférica derivada de las células de Schwann y la porción cubierta por mielina central derivada de la oligodendroglia $^{47}$.

La portio minor, o rama motora, sale de la protuberancia rostral y levemente medial a la zona de entrada de la portio major. Situada primero por delante de la raíz sensitiva, cerca de su borde superior, se coloca poco a poco por debajo de ella hasta alcanzar el cavum de Meckel. Discurre después medial e inferior al ganglio semilunar o de Gasser y acompaña finalmente a la rama mandibular del trigémino a través del foramen oval para inervar los músculos de la masticación. Se ha demostrado que del 10\% al 20\% de las fibras de la portio minor son amielínicas y tienen una función aferente o sensorial, de tal manera que pese a la sección aparentemente completa de la portio major, es posible que aún se preserve la sensibilidad facial.

La portio intermedia, o rama accesoria, está formada por un número variable de raicillas (entre una y diez) que discurren entre la portio major y la portio minor. Generalmente son ramificaciones de la portio major y entran en la protuberancia rostralmente en relación a la entrada de ésta. Se desconoce la función exacta de las fibras de la portio intermedia, aunque parece que podrían ser tanto fibras sensoriales como motoras que actuarían como vía accesoria y como intercomunicación entre las dos ramas principales ${ }^{18,47}$.

Tras su entrada en el puente, las fibras primarias aferentes del trigémino establecen conexiones sinápticas con uno de los varios núcleos del tronco cerebral. El haz de fibras que entra en la protuberancia y desciende caudalmente se llama tracto espinal trigeminal. Muchos de los axones de este tracto representan la única proyección central de muchas fibras de la rama sensorial. Según progresan caudalmente, el diámetro de estos axones va disminuyendo al mismo tiempo que pierden su cubierta mielínica y se enlentece su velocidad de conducción. Al descender, el tracto envía ramas a todos los niveles hacia el núcleo espinal trigeminal situado medialmente. Tanto el tracto como el núcleo espinal del trigémino se extienden hasta el nivel del segundo o tercer segmento cervical medular. Por otro lado, muchas fibras gruesas mielínicas de la portio major siguen un curso recto y corto hacia el núcleo sensorial o principal situado rostralmente a la entrada de la portio major en el puente ${ }^{18,47}$.

El complejo nuclear del trigémino en el tronco se subdivide según criterios citoarquitectónicos, funcionales y localizadores. El núcleo mesencefálico se considera relacionado fundamentalmente con la integración de la información propioceptiva de la mandíbula y dientes. El núcleo principal o sensorial situado en la protuberancia contiene sinapsis de fibras aferentes primarias que aportan sobre todo información sensitiva táctil. El núcleo espinal del trigémino se divide en tres subnúcleos: oralis, interpolaris y caudalis ${ }^{18}$. El caudalis es el más relacionado con la sensación dolorosa; su estructura es laminar e idéntica desde el punto de vista anatomofisiológico a la del asta posterior de la médula espinal. La función del subnúcleo interpolaris es dudosa. El subnúcleo oralis parece intervenir en la percepción de la sensibilidad oral y perioral, fundamentalmente nociceptiva.

Existen muchas conexiones intranucleares caudo-rostrales y rostro-caudales a lo largo del complejo nuclear trigeminal. De hecho, una gran proporción de neuronas de las capas profundas del subnúcleo caudalis no se proyectan al tálamo, sino que forman una vía intranuclear que podría estar involucrada en la modulación de componentes rostrales del complejo trigeminal del tronco encefálico. A su vez, los núcleos del trigémino mantienen conexiones con el tálamo, el colliculus superior, el cerebelo y con los núcleos de otros nervios craneales que intervienen en los reflejos orofaciales, como el núcleo del tracto solitario y el núcleo del hipogloso ${ }^{47}$.

\section{Etiopatogenia}

Para tratar de explicar el mecanismo a través del cual se produce una neuralgia idiopática se han postulado dos tipos de teorías: unas, evocan un origen central y otras, suponen que la causa de la lesión está en algún punto del trayecto nervio-ganglio-raíz.

Los estudios de Moses ${ }^{36}$, Beaver ${ }^{4}$ y Kerr $^{23,24}$ demuestran áreas de desmielinización segmentaria en la raíz trigeminal en pacientes con neuralgia esencial, lo cual apoya un mecanismo periférico como origen del dolor. La mielina aparece irregular, desorganizada, fragmentada, con aspecto degenerativo y en el interior, los axones están tortuosos, formando múltiples plegamientos. A través de estas áreas de desmielinización segmentaria, se podrían producir fenómenos de transmisión no sináptica que explicarían la existencia de fenómenos gatillo. De esta manera, un estímulo 
táctil transmitido a través de las fibras mielínicas podría ser desviado para activar las pequeñas fibras amielínicas produciendo dolor. El origen de la desmielinización segmentaria se debería a la acción mecánica compresiva que ejercen sobre la raíz posterior elementos vasculares en la zona de entrada de dicha raíz en la protuberancia. Para otros autores, la compresión mecánica se basaría en una excesiva angulación de la raíz en relación a modificaciones estructurales producidas durante el envejecimiento o a la presencia de bandas durales anómalas en el ápex del peñasco.

Por otra parte, $\mathrm{Cajal}^{8}$ había observado que en sujetos de edad avanzada las células ganglionares monopolares típicas de los ganglios sensitivos raquídeos y craneales, eran sustituidas parcialmente por formas neuronales atípicas entre las que destacaban células multipolares, fenestradas y deshilachadas. Curiosamente, este fenómeno de degeneración selectiva con sustitución por formas atípicas se veía también en el ganglio del glosofaríngeo, el cual está implicado en otra forma de neuralgia paroxística esencial, así como en estados patológicos diversos como la tabes, la esclerosis múltiple, los traumatismos y otros más, afecciones todas ellas que pueden dar lugar a síndromes dolorosos de perfil lancinante y paroxístico. Bajo estas circunstancias, los axones alcanzan proporciones extraordinarias con múltiples plegamientos que podrían facilitar el contacto entre las fibras y el desencadenamiento de la clínica característica.

Otros autores, abogan por un mecanismo central como causa de la neuralgia trigeminal idiopática ${ }^{13,14,25}$. Para ellos, los mecanismos periféricos por sí solos no son capaces de explicar los distintos fenómenos que se producen en la neuralgia trigeminal. Existiría, según estos autores, un fallo de los mecanismos centrales de inhibición que favorecería el desarrollo de los paroxismos dolorosos. Para Fromm y colaboradores $^{13,14}$, por ejemplo, el efecto terapéutico de la mayoría de los fármacos empleados en la neuralgia trigeminal depende precisamente de su capacidad para potenciar los mecanismos inhibitorios centrales. La irritación crónica en algún punto del trayecto desde la protuberancia hasta las terminaciones nerviosas conduce por una parte a una disminución de los mecanismos centrales de inhibición y por otra a la aparición de los potenciales de acción ectópicos en el nervio-raíz. La combinación de un aumento de actividad en la fibra aferente primaria con el empeoramiento de los mecanismos inhibitorios en el complejo nuclear trigeminal induce la aparición de descargas paroxísticas de las motoneuronas en el complejo nuclear trigeminal en respuesta a la estimulación táctil. Los fármacos útiles en el tratamiento de la neuralgia trigeminal actuarían, deprimiendo por un lado la actividad sináptica excitatoria en el núcleo espinal trigeminal, y por otro, facilitando la inhibición segmentaria a ese nivel.
King y colaboradores ${ }^{26}$ observaron que tres meses después de la administración de gel de alúmina en el núcleo y tracto espinal del trigémino en gatos y primates, se producía una respuesta exagerada a estímulos cutáneos en la hemicara afecta. Los animales presentaban una conducta de evitación ante cualquier estímulo en dicho territorio capaz de suprimir incluso la ingesta de alimentos. Los estudios electrofisiológicos demostraron la existencia de un potencial tardío centrífugo (de aproximadamente 10 milisegundos) en la raíz principal y en las distintas ramas después de la espiga inicial evocada por un estímulo cutáneo, que se originaba en el núcleo espinal trigeminal. El potencial de acción retardado en gatos no sometidos a la administración de alúmina, presentaba una amplitud del 10\% al 25\% del potencial de acción inicial, mientras que en los gatos tratados con gel de alúmina, este potencial de acción alcanzaba el $60 \%$ al $80 \%$ de amplitud del potencial inicial. Si se practicaba una compresión directa sobre el ganglio o sobre la raíz posterior, la conducta de evitación desaparecía al igual que lo hacía el potencial tardío de alto voltaje observado previamente sin que se produjese una clara hipoestesia facial. Posteriormente, Turnbull demostró que dicho potencial de acción tardío era de características antidrómicas. Se pensó, por tanto, que esto podría justificar la existencia de un círculo vicioso, de tal manera que el dolor que se producía después de un estímulo táctil se perpetuara por la conducción antidrómica desde el núcleo espinal trigeminal, no sólo a través de las fibras afectadas inicialmente sino también a través de otras reclutadas secundariamente. La compresión leve podría suprimir la conducción antidrómica sin afectar la conducción aferente lo que permitiría la conservación de la sensibilidad táctil.

Es probable que los mecanismos centrales participen en el desarrollo de la neuralgia trigeminal en aquellos casos en los que ya se ha establecido una lesión en las fibras aferentes primarias. Se han podido demostrar diversas modificaciones en el núcleo espinal trigeminal después de varias formas de deaferentización, con sobreexcitabilidad de los elementos neuronales y alteración tanto de los patrones de disparo como de la distribución somatotópica. De esta manera, parece posible construir una hipótesis fisiopatológica que combine adecuadamente las distintas observaciones anatomofuncionales mencionadas. Así, una lesión anatómica como la compresión vascular $\mathrm{u}$ otras formas de lesión mecánica sobre la raíz trigeminal, produciría áreas de desmielinización segmentaria que a su vez causaría modificaciones en el núcleo espinal trigeminal como se ha descrito en los modelos de deaferentización. La hiperactividad neuronal con patrones de disparo en salvas o ráfagas podría explicar el dolor espontáneo típico de la neuralgia trigeminal. Los fenómenos gatillo se deberían a la descarga neuronal excesiva que se produce en el núcleo espinal trigeminal tras estimulación periférica y después de deaferentización experimental, aunque también se 
explicarían por mecanismos de conducción o transmisión no sináptica. En definitiva, esta hipótesis mixta y de alguna manera ecléctica, contempla varios hechos clínicos observados en la neuralgia del trigémino. Asimismo, hace hincapié en diversas lesiones anatómicas macroscópicas como responsables o desencadenantes iniciales y también tiene en cuenta varios hechos histológicos, fisiológicos y farmacológicos observados en el laboratorio. Finalmente, integra mecanismos centrales y periféricos y considera la neuralgia del trigémino esencial como una forma de dolor por deaferentización.

\section{Tratamiento médico ${ }^{48}$}

1. El fármaco de elección es la carbamazepina: produce alivio completo o aceptable en el 69\% de los casos. Con el uso prolongado, la respuesta terapéutica disminuye en un $50 \%$ a pesar del incremento progresivo de la dosis administrada. Si se toleran 600 u $800 \mathrm{mg} /$ día y no se produce alivio, se deberá sospechar otro diagnóstico diferente al de neuralgia del trigémino ${ }^{43}$. Entre sus efectos secundarios se encuentra la somnolencia y la intolerancia gastrointestinal (las cuales se minimizan comenzando con dosis mínimas y aumentando éstas de manera lenta y progresiva); en muchos casos aparece una leucopenia relativa que normalmente no obliga a la retirada del fármaco; también puede aparecer diplopia transitoria, ataxia y otros.

Dosis: $100 \mathrm{mg}$, dos veces al día, oral; aumentos de 200 $\mathrm{mg}$ /día hasta un máximo de $1200 \mathrm{mg}$ /día repartidos en tres tomas.

En pacientes refractarios a la carbamazepina puede ser útil la oxcarbazepina, derivado estructural de aquélla aunque con menores efectos secundarios.

2. Baclofén: de $2^{\mathrm{a}}$ elección. Puede ser más efectivo si se administra junto a dosis bajas de carbamazepina. Posiblemente es teratogénico. Se debe evitar su retirada brusca ya que si no, puede causar alucinaciones y crisis convulsivas.

Dosis: comenzar con dosis bajas, $15 \mathrm{mg} /$ día repartidos en tres tomas, oral; aumentar cada tres días en $5 \mathrm{mg}$ cada una de las tres tomas sucesivamente, sin llegar a exceder $20 \mathrm{mg}$, cuatro veces al día (80 mg/día); se pautará la dosis efectiva más baja posible.

3. Pimozida: es un antipsicótico empleado principalmente en el síndrome de Gilles de la Tourette. No es un fármaco de primera línea en la neuralgia del trigémino. Puede ser útil en pacientes que no toleran la carbamazepina ${ }^{27}$. Prolonga el intervalo QT, por lo que no está indicado en el síndrome congénito de QT largo ni con otros fármacos que alarguen dicho intervalo.

Dosis: comenzar con $2 \mathrm{mg}$, dos veces al día, oral; aumentar cada cuatro días primero a $3 \mathrm{mg}$, dos veces al día, después a $4 \mathrm{mg}$, dos veces al día, y por último a un máximo de $6 \mathrm{mg}$, dos veces al día, según sea necesario para contro- lar los síntomas.

4. Fenitoína: puede ser útil vía intravenosa en pacientes que tienen tanto dolor que son incapaces de tomar la carbamazepina vía oral.

5. Capsaicina: $1 \mathrm{~g}$ aplicado tres veces al día durante varios días produjo remisión de los síntomas en 10 de 12 pacientes en un estudio; 4 de estos 10 recayeron en menos de 5 meses pero permanecieron sin dolor durante el resto del año de seguimiento del estudio después de una segunda aplicación del fármaco ${ }^{15}$.

6. Clonazepam: es útil en el $25 \%$ de los casos.

7. Amitriptilina: fármaco de gran interés al combinar sus propiedades analgésicas frente a dolores por deaferentización junto con las propias antidepresivas.

8. Otros: otros fármacos anticomiciales (gabapentina, lamotrigina...) se ha utilizan en la neuralgia del trigémino aprovechando sus propiedades frente a los dolores neuropáticos.

\section{Tratamiento quirúrgico}

El tratamiento quirúrgico de la neuralgia trigeminal se reserva para casos refractarios a la terapia médica o cuando los efectos secundarios de los fármacos empleados exceden los riesgos e inconvenientes de la cirugía ${ }^{42,48}$.

\section{Avulsión de ramas periféricas}

Comprende este apartado una serie de procedimientos que consisten en bloquear la rama periférica afecta por dolor o en bloquear las zonas trigger. Se puede realizar con alcohol, fenol o mediante neurectomía de la rama trigeminal afecta. La $1^{\mathrm{a}}$ rama (oftálmica) se bloquea en los nervios supraorbitarios o infraorbitarios; la segunda rama (maxilar) en el agujero redondo mayor; la tercera rama (mandibular) en el agujero oval o mediante neurectomía del nervio dental inferior. Técnicamente es un procedimiento sencillo, bien tolerado y que se puede realizar con anestesia local. Produce alivio del dolor que dura desde pocos meses hasta 1 ó 2 años $^{35}$. Fundamentalmente es un procedimiento paliativo, indicado principalmente en gente mayor o con mala situación clínica general.

\section{Técnicas quirúrgicas sobre la raíz trigeminal (micro- descompresión vascular, rizotomía trigeminal posterior y técnica de Spiller-Frazier)}

\section{Microdescompresión vascular}

Se basa en la teoría de que la neuralgia del trigémino se debe a la compresión de la raíz posterior por elementos vasculares anómalos ${ }^{19}$, principalmente la arteria cerebelosa superior ( $80 \%$ de los casos) y a veces por la arteria trigeminal primitiva persistente. Por otro lado, otros 
autores han demostrado compresión vascular de la raíz posterior hasta en un $50 \%$ de las autopsias de pacientes que no tenían neuralgia del trigémino.

A. Técnica: consiste en la realización de una craniectomía suboccipital paramediana. No entraré en detalles en lo que respecta a los aspectos quirúrgicos. Simplemente añadiré que independientemente del gesto quirúrgico de separar la arteria de la raíz nerviosa con un parche de teflón o similar lo más próximo al tronco cerebral, la maniobra de tocar reiteradamente la raíz trigeminal permite aliviar el dolor de forma característica. Curiosamente, esta forma de "lesionar" o "deaferentizar" la raíz mejora el cuadro doloroso contraviniendo de alguna manera la hipótesis fisiopatológica de que la neuralgia del trigémino corresponde a una forma de dolor por deaferentización. En contra de lo defendido por Jannetta, parece poco probable que las venas dada su ausencia de pulsatilidad, sean responsables de la compresión de la raíz trigeminal.

B. Resultados ${ }^{1,7}$ : la microdescompresión vascular ofrece resultados aceptables, de tal manera que en el 75\% al $80 \%$ de los pacientes el resultado puede calificarse de exitoso; en un $10 \%$ adicional el resultado es bueno aunque no se consigue un alivio total; por tanto, su tasa de fracaso inicial está alrededor del 10\%. El éxito de este procedimiento se relaciona con la duración previa de la clínica: cuando la evolución de los síntomas es superior a los ocho años, la proporción de enfermos que obtienen alivio inicial disminuye significativamente. De tal manera, que cuanto más tiempo espera uno para realizar una microdescompresión vascular, más bajo será el porcentaje de éxito que se obtendrá. Para algunos autores, este tiempo no ha de ser superior a los dos años. Por otro lado, hay que resaltar que resulta menos efectiva en pacientes con las tres ramas del trigémino afectas o en aquéllos que fueron sometidos con anterioridad a un procedimiento destructivo.

La proporción de recurrencias es difícil de conocer; en una serie de 40 pacientes seguidos durante ocho años y medio se obtuvieron los siguientes resultados ${ }^{7}$ : el promedio de recurrencia mayor (dolor recurrente no controlado con fármacos) fue del $31 \%$; el promedio de recurrencia menor (dolor recurrente leve o que se controla con medicación) fue del 17\%; utilizando la curva de Kaplan-Meier, se espera que el $80 \%$ de los pacientes esté libre de dolor o tenga recurrencia menor a los 5 años y el $70 \%$ a los 8 años y medio (por tanto, el índice de recurrencia es de un 5\% cada año, aproximadamente); aquellos pacientes en los que se observó compresión venosa del nervio en el campo quirúrgico tuvieron más recurrencias que aquellos otros en los que se objetivó una compresión arterial importante; este estudio no encontró correlación entre cirugía destructiva previa y promedio de recurrencia mayor.

C. Efectos secundarios y complicaciones ${ }^{17,20,40}$ : teóricamente, la microdescompresión vascular no debe ocasionar déficit sensorial postquirúrgico, aunque la manipulación de la raíz puede provocar cierta disestesia en una proporción baja de pacientes que raramente o nunca llega al grado de anestesia dolorosa. Así pues, la incidencia de anestesia facial es mucho menor que con los procedimientos percutáneos. De todas maneras, se puede observar un déficit sensorial leve en el $25 \%$ de los pacientes.

Al tratarse de una cirugía de fosa posterior, la mortalidad se sitúa entre el 0,22\% y el $2 \%$ en manos experimentadas.

En cuanto a la morbilidad cabe destacar lo siguiente: en el $20 \%$ de los casos se produce una meningitis aséptica, que normalmente debuta tres a siete días después de la realización del procedimiento, y que clínicamente se manifiesta como dolor de cabeza, meningismo, pleocitosis y cultivo de LCR negativo, respondiendo a los esteroides y a las punciones lumbares; aparece morbilidad neurológica mayor en el $1 \%$ al $10 \%$ de los casos (promedios que son mayores en neurocirujanos menos experimentados), incluyendo: sordera (1\%), disfunción del nervio vestibular y disfunción del nervio facial; parálisis de nervios craneales: del IV par craneal produciendo diplopia (en el 4,3\%, siendo la mayoría transitorias), del VIII (en el 3\% de los casos, provocando pérdida de audición) y del VII (en el 1,6\%, siendo también la mayoría transitorias); hemorragias postoperatorias, incluyendo hematoma subdural, intracerebral y hemorragia subaracnoidea; crisis convulsivas y estado epiléptico e infarto cerebral en el territorio de la arteria cerebral posterior o en el tronco cerebral.

D. Indicaciones generales ${ }^{32}$ : podemos resumir las indicaciones generales de la microdescompresión vascular en tres apartados:

a) pacientes menores de 70 años que padecen neuralgia trigeminal, con una supervivencia estimada de 5 años o más, sin factores de riesgo médicos o quirúrgicos significativos (aunque la exploración quirúrgica de la fosa posterior es normalmente bien tolerada, la morbilidad quirúrgica aumenta con la edad, por lo que esta técnica no está indicada en pacientes por encima de los 70 años).

b) puede emplearse en pacientes que no se ajustan estrictamente a los criterios expuestos en a) pero que tienen dolor intratable pese a haberse sometido a procedimientos percutáneos previos.

c) neuralgias de la $1^{\mathrm{a}}$ rama en enfermos para quienes el riesgo de queratitis por exposición debida a anestesia corneal sea inaceptable (por ejemplo, pacientes con ceguera en el ojo contralateral) o en aquellos sujetos que deseen evitar la anestesia facial por cualquier razón.

Como ya se comentó con anterioridad, aproximadamente el $2 \%$ de los pacientes con esclerosis múltiple tendrán una placa desmielinizante en la zona de entrada de la raíz trigeminal que les causa dolor neurálgico y que habitualmente no responde a la microdescompresión vascular, 
por lo que las técnicas percutáneas son el procedimiento de elección en ellos.

\section{Rizotomía trigeminal posterior}

Fue introducida por Dandy en 1932, quien observó que con esta técnica se aliviaba el dolor neurálgico con pérdida disociada de la sensibilidad (afectación mayor del componente algésico y en menor medida de la sensibilidad táctil). Consiste en la sección de la mitad o los dos tercios inferiores de la portio major de la raíz trigeminal, aprovechando su organización somatotópica (cerca de la protuberancia las fibras de V1 quedan superiores y las de V3 inferiores) ${ }^{42}$.

El principal problema que conlleva esta técnica es que no se puede controlar el defecto sensorial que se va a producir. Además, existe riesgo de causar anestesia dolorosa o de provocar la abolición del reflejo corneal. Por otro lado, es una cirugía mayor con las complicaciones que esto supone, al igual que la microdescompresión vascular.

Actualmente es una técnica que prácticamente sólo se realiza a pacientes en los que al intentar una microdescompresión vascular, no se observan hallazgos relevantes en el campo quirúrgico (no se encuentra contacto vascular con el nervio o no se encuentra deformidad anatómica del propio nervio), así como también como medida de último recurso en enfermos que padecen neuralgia recurrente a pesar de haberse sometido a uno o varios procedimientos percutáneos en presencia de anestesia facial total. En el caso de pacientes con anestesia facial preoperatoria o cuando se sospeche por cualquier razón que la conducción del dolor puede estar realizándose a través de vías alternativas, se debería considerar la sección de la rama motora (portio minor) como vía alternativa de conducción de los estímulos dolorosos, además de la sección de la portio major $^{22}$. Asimismo, puede ser útil en pacientes que han sufrido varias microdescompresiones vasculares repetidas o en aquellos enfermos cuyos síntomas superan los ocho o nueve años de duración, ya que como se ha dicho anteriormente este grupo de sujetos obtienen porcentajes de éxito inferiores cuando se emplea la microdescompresión vascular como único procedimiento 5 .

\section{Técnica de Spiller-Frazier}

Dentro del grupo de técnicas quirúrgicas sobre la raíz trigeminal debemos incluir este procedimiento, que consiste en una rizotomía retrogasseriana mediante aproximación subtemporal extradural. Rara vez se emplea en la actualidad $^{42}$.

\section{Tractotomía bulbar trigeminal}

Basada en las observaciones de Hum quien describió el caso de un paciente con analgesia completa de la hemicara, de inicio súbito, sin afectación de la sensibilidad táctil. Al
2010; 21: 361-372

realizarse la autopsia de este paciente, se descubrió un área de reblandecimiento de origen isquémico 7-8 $\mathrm{mm}$ dorsal a la porción medial de la oliva inferior. Aprovechando estos datos, Sjövist eligió dicho punto para realizar una incisión a ese nivel. También se utilizó la estereotaxia para practicar una lesión en esa localización.

Es un procedimiento en desuso dado que tiene una elevada incidencia de complicaciones, muchas de ellas permanentes ${ }^{42}$. No obstante, se han obtenido resultados aceptables en pacientes refractarios a cualquier otra terapia, mediante termocoagulación por radiofrecuencia del subnúcleo cuadalis tras exposición quirúrgica del mismo9.

\section{Rizotomía percutánea con glicerol}

Consiste en inyectar un volumen de glicerol anhidro al 99,9\% en la cisterna del cavum de Meckel $^{16}$. La cisternografía con metrizamida empleada en la descripción original del procedimiento no es esencial. Es una técnica sencilla de realizar. Aunque también se ha empleado alcohol, el glicerol tiene mejores resultados.

Obtiene alivio sintomático en un $90 \%$ de los pacientes con baja incidencia de defecto sensorial residual. Posiblemente tiene una incidencia más baja de hipoestesia y anestesia dolorosa que la termocoagulación por radiofrecuencia $^{31}$.

Su principal inconveniente estriba en su elevada tasa de recurrencia, ya que a largo plazo menos del $50 \%$ de los pacientes tratados disfrutan de una buena analgesia ${ }^{12}$. La repetición del procedimiento incrementa el riesgo de provocar defecto sensorial residual y, por tanto, de disesetesia y anestesia dolorosa.

\section{Termocoagulación diferencial por radiofrecuencia del ganglio de Gasser}

Se basa en la hipótesis inicial que suponía que las fibras C (amielínicas) y las A finamente mielinizadas (delta), que transmiten la sensibilidad dolorosa, deben ser más vulnerables a la destrucción por elevación térmica controlada $\left(70^{\circ} \mathrm{C}\right)$ que las fibras gruesamente mielinizadas, las cuales transmiten la sensibilidad propioceptiva y la táctil (A alfa y A beta, respectivamente). Esta destrucción selectiva permitiría, por tanto, la preservación de la sensibilidad táctil y la desaparición del dolor. Actualmente se ha demostrado mediante diversos estudios experimentales que la termocoagulación afecta prácticamente por igual a todos los tipos de fibras.

A. Técnica: tal como se utiliza en la actualidad fue desarrollada por Sweet y Wepsic ${ }^{44}$. El lugar de punción está 2,5 ó $3 \mathrm{cms}$. lateral a la comisura bucal. Se dirige el trócar bajo fluoroscopia y sedación hacia la intersección del ápex del peñasco con el clivus, medial al proceso coronoide 
mandibular. Debe asegurarse la posición correcta del trócar, evitando su inserción en la fisura orbitaria superior (podría producirse ceguera) o en el agujero redondo menor (por donde transcurre la arteria meníngea media). Si fuera necesario visualizar el agujero oval por existir dificultad en su canalización, se puede identificar éste extendiendo el cuello $20^{\circ}$ y rotando la cabeza unos $15-20^{\circ}$ al lado contrario de la lesión. Ha de monitorizarse la tensión arterial durante todo el procedimiento ya que la inserción de la aguja y/o la propia lesión como tal, causan a menudo hipertensión arterial. Una vez canalizado el foramen oval, se retira el trócar y se inserta el electrodo. A continuación se despierta al paciente y se inducen parestesias con la estimulación eléctrica de bajo voltaje. Existe en el mercado un electrodo recto y uno curvo. Teóricamente, el electrodo curvo permite ser selectivo sobre una de las tres ramas terminales del nervio trigémino. Cuando las parestesias reproducen la distribución del tic doloroso, se provoca la lesión térmica (bajo anestesia nuevamente, a 60-70 durante 90 segundos, para el primer intento), apareciendo el característico enrojecimiento facial. En el caso de que el paciente no sea capaz de percibir dichas parestesias por padecer alteraciones de la sensibilidad facial secundarias a lesiones previas, se estimulará con frecuencias distintas y se observará la contracción del masetero (esto requiere, al menos, la preservación de la portio minor o motora). Seguidamente, se comprobará que la hipoalgesia es satisfactoria; en caso de que no lo fuera, se repetirá el procedimiento. Además, se realizará una exploración de la sensibilidad facial en todas las ramas trigeminales, incluyendo el reflejo corneal bilateral; asimismo, se explorará la musculatura ocular extrínseca, la función de los maseteros (con la boca cerrada, se palpan las mejillas para ver la contracción de dichos músculos) y la de los pterigoideos (al abrir la boca, el mentón se desvía hacia el lado enfermo, en caso de paresia).

$B$. Resultados: el éxito terapéutico se consigue en más del 95\% de los casos, pero la incidencia de disestesias y anestesia dolorosa postoperatorias son superiores a las observadas con otros procedimientos ${ }^{39}$. En caso de neuralgia de $1^{\text {a }}$ rama, la incidencia de anestesia corneal es además muy elevada (aproximadamente 4\%) lo cual contraindica esta técnica para aquellas neuralgias que tienen dicha localización.

C. Efectos secundarios y complicaciones: prácticamente siempre ocurre algún grado de adormecimiento facial, tanto en éste como en otros procedimientos percutáneos cuyo resultado terapéutico se califica de exitoso y por tanto, no se considera una complicación como tal.

Solamente se han descrito 17 fallecimientos de un total de más de 22.000 procedimientos (incluyendo los realizados por neurocirujanos menos experimentados y pacientes con elevado riesgo quirúrgico $)^{43}$.

En lo que respecta a la morbilidad, destaco lo siguiente $^{34,45}$ : parestesias (del 11\% al 93\% de los pacientes); debilidad parcial del masetero (normalmente no percibido por el paciente, del 7\% al 50\% de los casos); disestesias (pueden ser menores (13\% de los casos) o llegar al rango de anestesia dolorosa (dolor quemante, constante, de elevada intensidad, sin respuesta efectiva a ningún tratamiento, en el 2\% al 4\% de los casos); en general, la proporción de disestesias es más elevada cuanto más completas son las lesiones); disminución de la audición (del 0\% al 27\% de los pacientes, secundaria a paresia del tensor del tímpano, también conocido como músculo del martillo, inervado por la parte motora del $\mathrm{V}$ par craneal); paresia oculomotora (habitualmente transitoria, del $0 \%$ al 20\% de los casos); en el $20 \%$ de los pacientes se producen alteraciones en el lagrimeo (aumento en el 17\% y descenso en el $3 \%$ ); en otro $20 \%$, las alteraciones se dan en la salivación (aumentando también en torno al 20\% y disminuyendo en el 3\%); queratitis neuroparalítica (por alteración de sensibilidad corneal, del $0 \%$ al 4\% de los casos); meningitis (en el 0,3\% de los pacientes); siete casos de hemorragia intracraneal (seis de ellos, fatales) en más de 14.000 procedimientos, probablemente debido a hipertensión arterial transitoria (presión arterial sistólica cercana a los $300 \mathrm{mmHg}$ ) que debe tratarse con nitroprusiato sódico; otras complicaciones más raras incluyen la fístula carótido-cavernosa, el absceso del lóbulo temporal y la meningitis aséptica.

Algunas de estas complicaciones, como las parestesias, la debilidad parcial del masetero, la disminución de la audición, la paresia oculomotora o la queratitis neuroparalítica son más frecuentes cuando se usa el electrodo recto que cuando se utiliza el curvo, por lo que éste es el que ha de usarse preferentemente.

\section{Compresión percutánea del ganglio de Gasser (Mullan- Lichtor)}

Este procedimiento es una adaptación de la compresión trigeminal directa por vía subtemporal del ganglio de Gasser que realizó Shelden en 1955. Este autor observó que el común denominador de las técnicas descompresivas realizadas hasta entonces era precisamente la lesión mecánica que se ejercía sobre el ganglio o la raíz mientras se intentaba liberar quirúrgicamente alguna de estas dos estructuras $^{41}$. El procedimiento percutáneo tal y como se realiza hoy día, fue introducido por Mullan y Lichtor en 1978 y publicado por estos mismos autores en $1983^{38}$.

A. Técnica ${ }^{38}$ : consiste en introducir un balón de Fogarty en el cavum de Meckel a través de un trócar que penetra en el foramen oval. El inflado del balón bajo visión radioscópica permite una compresión ganglionar controlada. Los pacientes son premedicados para inducir una sedación suave antes de pasar al quirófano. Se realiza una anestesia de corta duración. No es precisa la intubación 
orotraqueal en la mayoría de los casos. El paciente es colocado en decúbito supino sobre la mesa de quirófano con la cabeza en posición neutra. Se utiliza un fluoroscopio para control de la punción. El punto de entrada en la piel se sitúa $2,5 \mathrm{~cm}$. externo a la comisura bucal y $0,5 \mathrm{~cm}$. por encima de la misma. Se introduce un trócar del n ${ }^{\circ} 14$, que se dirige primeramente paralelo al plano sagital para evitar atravesar la mucosa de la cavidad oral y posteriormente medial, hacia el foramen oval, evitando la penetración más allá de los límites de esta estructura. La técnica de punción remeda la utilizada para la termocoagulación del ganglio de Gasser. Posteriormente, se retira el fiador y se introduce un catéter de Fogarty del $n^{\circ} 4$, cuya punta debe sobrepasar entre 10 y $12 \mathrm{~mm}$ la punta del trócar. Después, se procede al inflado del balón con material de contraste, habitualmente iopamidol, hasta un volumen medio de 0,7 a $0,75 \mathrm{ml}$. La forma y posición del balón se comprueba en relación con las estructuras vecinas (clivus, silla turca y borde superior del peñasco). Si la posición del balón no es correcta, se deshincha éste y se recoloca el trócar hasta adquirir la posición correcta. La morfología más adecuada del balón distendido debe ser esférica u oval, con una protrusión a nivel posterior que corresponde a su herniación parcial a través del porus trigemini adoptando así la clásica disposición piriforme, garantía de que el balón está adecuadamente posicionado. El volumen del balón que se desplaza hacia la fosa posterior puede variar apareciendo a veces formas en reloj de arena, que también son correctas terapéuticamente. El inflado del balón fuera del cavum de Meckel da lugar a formas cilíndricas, parecidas a las que se producen in vitro, o a formas irregulares que no conducen a un resultado funcional satisfactorio. Si es el primer procedimiento de este tipo que se realiza al enfermo, el inflado del balón se mantiene durante 1 minuto; en pacientes con dolor recurrente intratable, el inflado puede mantenerse hasta 3 minutos. Posteriormente, se procede a aspirar el material de contraste y a retirar el catéter y el trócar. Sobre el punto de punción cutáneo se realiza compresión manual durante unos minutos para prevenir la formación de hematomas. La duración total del procedimiento no suele ser superior a 15 ó 20 minutos. El paciente es dado de alta en las 24 horas siguientes si no aparece ninguna complicación.

B. Resultados ${ }^{12,30,33}$ : se cree que los efectos terapéuticos se deben al daño mecánico y a la isquemia inducidos por el balón sobre las fibras nerviosas y las células ganglionares. El promedio de alivio inmediato es del $89,9 \%$ al $100 \%$. Lichtor y Mullan publicaron un $80 \%$ de éxito a los 5 años y un $70 \%$ a los 10 años de seguimiento ${ }^{28}$.

La recurrencia total varía ampliamente, desde 54\% a $74 \%$ a los 3 años para algunos autores, al 30\% a los 10 años para otros. En los pacientes que padecen recidiva de la sintomatología dolorosa, se puede reiniciar el tratamiento con carbamazepina, existiendo por lo general buena res- puesta con la administración de dosis bajas ${ }^{29}$. En caso de que no exista una respuesta adecuada al fármaco, se puede repetir la compresión percutánea.

El promedio de reintervención a los cinco años es del $13 \%$, que es un porcentaje mejor que el de la termocoagulación percutánea $(21 \%$ a $28 \%)$ o el de la microdescompresión vascular (17\% a 26\%). Pese a este aparente éxito, el $15 \%$ de los pacientes con expectativa de vida mayor de 10 años requerirán algún tipo de cirugía más invasiva con posterioridad.

La duración de la compresión se relaciona con el déficit sensitivo postoperatorio y éste es directamente proporcional al tiempo que el paciente permanecerá sin dolor, de tal manera que la ausencia de hipoestesia tras el procedimiento es predictivo de alivio del dolor durante un corto período de tiempo.

C. Efectos secundarios y complicaciones ${ }^{28,30,33}$ : es importante subrayar que la mortalidad del procedimiento es muy rara.

En lo referente a la morbilidad señalo lo siguiente: un grado leve de hipoestesia en el territorio de la $3^{\mathrm{a}}$ rama trigeminal es la regla aunque la mayoría de los pacientes se acostumbran bien a ella; el 30\% de los pacientes refieren parestesias postoperatorias en la distribución de una o más ramas trigeminales; del 3,5\% al 8,5\% desarrollan disestesia facial que exige tratamiento (la mayor incidencia de disestesias postoperatorias se produce en aquellos casos en los que el balón adopta forma de reloj de arena; la frecuencia de estas disestesias es baja si se compara con la de otros procedimientos percutáneos; la aparición de anestesia dolorosa es sumamente infrecuente ${ }^{12}$ ); debilidad de los músculos masticatorios ipsilaterales (casi todos los pacientes presentan alguna debilidad del masetero ipsilateral que normalmente desaparece en los 3 meses siguientes pero puede durar hasta 1 año; se constata debilidad significativa del masetero en el $1 \%$ de los sujetos inicialmente, con un $3 \%$ de frecuencia permanente); también se puede producir fallo en el inflado del balón y otros problemas de índole técnico; erupciones herpéticas; parálisis del VI par craneal (3-5\%); hematoma en la mejilla; meningitis aséptica $(5 \%$; está descrita la meningitis por neumococo secundaria a la transgresión de la mucosa oral durante la canulación del foramen oval ${ }^{30}$; otorragia transitoria $(5 \%)$; la queratitis y la pérdida del reflejo corneal son raras porque la aguja queda en íntimo contacto con las fibras de la $3^{a}$ rama del trigémino (V3) que son inferolaterales, soslayando las fibras que forman la $1^{\mathrm{a}}$ rama (V1) que quedan superomediales ${ }^{7}$; finalmente, complicaciones vasculares (fístula carótidocavernosa, fístula arterio-venosa dural y diversos tipos de hemorragia intracraneal (hemorragia subaracnoidea, hematoma subdural y hematoma intraparenquimatoso)) $)^{2}$.

La compresión del ganglio de Gasser induce dos fenómenos perioperatorios bien $\operatorname{conocidos}^{30}$ : por un lado 
bradicardia y por el otro, una elevación brusca de la tensión arterial, que puede requerir nitroprusiato sódico para su control, mediada parece ser por una respuesta simpatoadrenal originada por la estimulación mecánica de las terminaciones nerviosas de la duramadre del cavum de Meckel o del propio ganglio de Gasser. La lidocainización del cavum previa a la compresión parece ser la única maniobra capaz de inhibir esta liberación masiva de catecolaminas ${ }^{10}$. Existe controversia sobre si la bradicardia en estos casos es significativa o no. Algunos autores usan rutinariamente marcapasos externos y atropina para frecuencias cardíacas por debajo de 45 latidos por minuto. Otros administran atropina intramuscular preoperatoriamente e intravenosa perioperatoriamente para los episodios de bradicardia durante la canulación. Finalmente, otros no tratan estos episodios, normalmente autolimitados, y los usan como indicador para saber si el foramen oval ha sido canalizado y si después se ha logrado una compresión adecuada.

D. Comparación con otras técnicas percutáneas ${ }^{12}$ : las principales ventajas de esta técnica son las siguientes: fácil y rápida de realizar; no precisa cooperación por parte del paciente a diferencia de otros procedimientos percutáneos; soporta una baja incidencia de disestesias y de anestesia corneal postoperatorias y tiene una proporción de recidivas moderada. Para algunos autores, los porcentajes de disestesias y recurrencias de la técnica de MullanLichtor son comparables a los de la termocoagulación por radiofrecuencia o a los de la rizotomía percutánea con glicerol.

Sus principales inconvenientes radican en que las lesiones no pueden ser confinadas a la distribución de una única rama del trigémino; al menos, requiere de anestesia general ligera (aunque se ha conseguido realizar todo el procedimiento con anestesia local mediante infiltración con lidocaína del lugar de punción y del propio ganglio de Gasser ${ }^{10}$ y se precisa un trócar del $n^{0} 14$, que es más grande que el del $n^{\circ} 20$ usado en la termocoagulación por radiofrecuencia y en la rizotomía percutánea con glicerol, con lo cual la canulación del foramen oval es más difícil que con estas dos técnicas ${ }^{30}$. Algunos autores también consideran que la hipertensión arterial y la bradicardia intraoperatorias son una desventaja pero, de hecho, la incidencia de hipertensión intraoperatoria es menor con la compresión percutánea que con la radiofrecuencia. Sin embargo, la bradicardia y la parálisis de la raíz motora trigeminal ipsilateral son más frecuentes con la compresión percutánea, de tal manera que esta técnica está contraindicada si ya existe una parálisis contralateral de los músculos masticatorios por un procedimiento previo.

E. Indicaciones generales ${ }^{28,37}$ : la técnica de MullanLichtor está especialmente indicada en la neuralgia de la $1^{a}$ rama del trigémino dada la baja tasa de anestesia corneal observada respecto a otras técnicas. También es aplicable a enfermos en mal estado general o a pacientes que sobrepasan los 70 años de edad, indicaciones compartidas con la termocoagulación por radiofrecuencia. Asimismo, son subsidiarios de la compresión percutánea aquellos pacientes sometidos a técnicas destructivas previamente y que quedaron sin disestesias faciales importantes. Finalmente, la termocoagulación por radiofrecuencia puede ser una alternativa válida a la compresión percutánea para aquellos enfermos que presentan neuralgia en el territorio de la $3^{\mathrm{a}}$ rama trigeminal.

\section{Manejo de los fracasos terapéuticos}

El 90\% de las recurrencias ocurren en la misma distribución de las ramas previamente afectas; en el restante $10 \%$, estas recurrencias aparecen en una nueva división trigeminal y pueden representar una progresión del proceso subyacente.

Los procedimientos percutáneos pueden repetirse en pacientes que sufren una recurrencia y que preservan parte de la sensibilidad facial. La repetición de dicho procedimiento percutáneo es a menudo efectiva.

Cuando las técnicas percutáneas definitivamente fracasan, se puede realizar una microdescompresión vascular pero la proporción de éxito de esta última puede verse reducida, siendo del 90\% aproximadamente en pacientes a quienes se les realiza por primera vez dicho procedimiento y del $43 \%$ para aquellos a quienes se les hace una microdescompresión vascular tras una técnica percutánea (debe considerarse, no obstante, que este porcentaje del $90 \%$ puede estar sobrevalorado y también debe tenerse en cuenta que el grupo de pacientes en quienes fallaron los procedimientos percutáneos puede seleccionar un subgrupo de enfermos cuya neuralgia es más difícil de tratar $)^{3}$.

La microdescompresión vascular también puede repetirse en pacientes que padecen una recurrencia tras una primera microdescompresión, teniendo en cuenta que el material que se interpone entre la estructura vascular y el nervio ha podido dejar de ejercer su función por resbalar o por cualquier otro motivo ${ }^{11}$. También puede haber sucedido que el vaso que realmente causa la neuralgia trigeminal haya sido separado del nervio por la posición intraoperatoria y por tanto, se haya atribuido el origen del dolor neurálgico a una estructura vascular que en realidad no motivaba dicho dolor. Por este motivo, algunos autores preferimos la posición sentada al "park bench" para la realización de esta técnica, ya que de alguna manera la disposición anatómica de las estructuras neurovasculares queda menos distorsionada con la primera.

Finalmente, dedicaré unas breves palabras a la radiocirugía. Hasta hace poco tiempo, esta técnica se reservaba de alguna manera para pacientes que no habían respondido a ninguno de los tratamientos previamente descritos. 
Hoy día, se sabe que los resultados de este procedimiento terapéutico son muy buenos a corto plazo. Sin embargo, las recurrencias pueden alcanzar el $50 \%$ a largo plazo (5 años de seguimiento), particularmente en pacientes que han sufrido tratamientos invasivos previos. Esta circunstancia obliga, por tanto, a reconsiderar la indicación de la radiocirugía como opción terapéutica de último recurso.

\section{Bibliografía}

1. Alberione, F., Arena, A., Matera, R.: [Microvascular descompression for trigeminal neuralgia: prognostic [corrected] factors]. Neurocirugia 2008; 19: 242-247.

2. Arrese, I., Lobato, R.D., Alén, J.F., Lagares, A., Miranda, P.: Acute subdural and intratemporal hematoma as a complication of percutaneous compression of the gasserian ganglion for trigeminal neuralgia. Neurocirugia 2005; 16: 177-182.

3. Barba, D., Alksne, J.F.: Success of microvascular decompression with and without prior surgical therapy for trigeminal neuralgia. J Neurosurg 1984; 60: 104-107.

4. Beaver, D.L.: Electron microscopy of the gasserian ganglion in trigeminal neuralgia. J Neurosurg 1967; 26: S138150.

5. Bederson, J.B., Wilson, C.B.: Evaluation of microvascular decompression and partial sensory rhizotomy in 252 cases of trigeminal neuralgia. J Neurosurg 1989; 71: 359-367.

6. Bullitt, E., Tew, J.M., Boyd, J.: Intracranial tumors in patients with facial pain. J Neurosurg 1986; 64: 865-871.

7. Burchiel, K.J., Clarke, H., Haglund, M., Loeser, J.D.: Long-term efficacy of microvascular decompression in trigeminal neuralgia. J Neurosurg 1988; 69: 35-38.

8. Cajal, S.R.: Degeneration and regeneration of the sensory and sympathetic ganglia. En May RM (ed): Degeneration and regeneration of the nervous system. London; Oxford University Press, 1928, Vol II; pp. 397-413.

9. Delgado-López, P., García-Salazar, F., Mateo-Sierra, O., Carrillo-Yagüe, R., Llauradó, G., López, E.: Trigeminal nucleus caudalis dorsal root entry zone radiofrequency thermocoagulation for invalidating facial pain. Neurocirugia 2003; 14: 25-32.

10. Domínguez, J., Lobato, R.D., Rivas, J.J., et al: Changes in systemic blood pressure and cardiac rhythm induced by therapeutic compression of the trigeminal ganglion. Neurosurgery $1994 ; 34: 422-428$.

11. Fernández-Carballal, C., García-Salazar, F., PérezCalvo, J., García-Leal, R., Gutiérrez, F.A., Carrillo, R.: [Management of recurrent trigeminal neuralgia after failed microvascular decompression]. Neurocirugia 2004; 15 : 345352.

12. Fraioli, B., Esposito, V., Guidetti, B., Cruccu, G., Manfredi, M.: Treatment of trigeminal neuralgia by thermocoagulation, glycerolization and percutaneous compression of the gasserian ganglion and/or retrogasserian rootlets: long term results and therapeutic protocol. Neurosurgery 1989; 24 : 239-245.

13. Fromm, G.H., Chattha, A.S., Terrence, C.F., Glass, J.D.: Role of inhibitory mechanisms in trigeminal neuralgia. Neurology 1981; 31: 683-687.

14. Fromm, G.H., Terrence, C.F., Maroon, J.C.: Trigeminal neuralgia. Current concepts regarding etiology and pathogenesis. Arch Neurol 1984; 41: 1204-1207.

15. Fusco, B.M., Alessandri, M.: Analgesic effect of capsaicin in idiopathic trigeminal neuralgia. Anesth Analg 1992; 74: 375-377.

16. Hakanson, S.: Trigeminal neuralgia treated by the injection of glycerol into the trigeminal cistern. Neurosurgery 1981; 9: 638-646.

17. Hanakita, J., Kondo, A.: Serious complications of microvascular decompression operations for trigeminal neuralgia and hemifacial spasm. Neurosurgery 1988; 22: 348352.

18. Humphrey, T.: The central relations of trigeminal nerve. En Kahn EA, Crosby EC, Schneider RC, Taren JA (eds). Correlative neurosurgery, $20^{\text {th }}$ ed. Springfield; Charles C Tomas, 1973; pp. 477-492.

19. Jannetta, P.J.: Arterial compression of the trigeminal nerve at the pons in patients with trigeminal neuralgia. J Neurosurg 1967; 26: S159-162.

20. Jannetta, P.J.: Microsurgical management of trigeminal neuralgia. Arch Neurol 1985; 42: 800.

21. Katusic, S., Beard, C.M., Bergstralh, E., Kurland, L.T.: Incidence and clinical features of trigeminal neuralgia, Rochester, Minnesota, 1945-1984. Ann Neurol 1990; 27: 89-95.

22. Keller, J.T., van Loveren, H.: Pathophysiology of the pain of trigeminal neuralgia and atypical facial pain: a neuroanatomical perspective. Clin Neurosurg 1985; 32: 275-293.

23. Kerr, F.W.: Pathology of trigeminal neuralgia: light and electron microscopic observations. J Neurosurg 1967; 26: S151-156.

24. Kerr, F.W.: Evidence for a peripheral etiology of trigeminal neuralgia. J Neurosurg 1967; 26: S168-174.

25. King, R.B.: Evidence for a central etiology of tic douloureux. J Neurosurg 1967; 26: S175-180.

26. King, R.B., Meagher, J.N., Barnett, J.C.: Studies of trigeminal nerve potentials in normal compared to abnormal experimental preparations. J Neurosurg 1956; 13: 176-183.

27. Lechin, F., van der Dijs, B., Lechin, M.E., et al.: Pimozide therapy for trigeminal neuralgia. Arch Neurol 1989; 46: 960-963.

28. Lichtor, T., Mullan, J.F.: A 10-year follow-up review of percutaneous microcompression of the trigeminal ganglion. $\mathrm{J}$ Neurosurg 1990; 72: 49-54.

29. Lobato, R.D., Rivas, J.J., Sarabia, R.: Percutaneous compression of the gasserian ganglion for trigeminal neuralgia. En Mumenthaler M, van Zwieten PA, Farcot JM (eds). Treatment of chronic pain: possibilities, limitations and long 
term follow-up. London; Harwood Academic Publishers, 1990; pp. 215-230.

30. Lobato, R.D., Rivas, J.J., Sarabia, R., Lamas, E.: Percutaneous microcompression of the gasserian ganglion for trigeminal neuralgia. J Neurosurg 1990; 72: 546-553.

31. Lunsford, L.D.: Percutaneous retrogasserian glycerol rhizotomy. En Rovit RL, Murali R, Jannetta PJ (eds). Trigeminal neuralgia. Baltimore; Williams and Wilkins, 1990; pp. $145-164$.

32. Lunsford, L.D., Apfelbaum, R.I.: Choice of surgical therapeutic modalities for treatment of trigeminal neuralgia: microvascular decompression, percutaneous retrogasserian thermal, or glycerol rhizotomy. Clin Neurosurg 1985; 32: 319333.

33. Meglio, M., Cioni, B.: Percutaneous procedures for trigeminal neuralgia: microcompression versus radiofrequency thermocoagulation. Pain 1989; 38: 9-16.

34. Menzel, J., Piotrowski, W., Penzholz, H.: Long-term results of Gasserian ganglion electrocoagulation. J Neurosurg 1975; 42: 140-143.

35. Morley, T.P.: General considerations, medical therapy and minor operative procedures for trigeminal neuralgia. En Youmans JR (ed). Neurological Surgery, $3^{\text {rd }}$ ed. Philadelphia; WB Saunders, 1990; pp. 3880-3887.

36. Moses, H.L.: Comparative fine structure of the trigeminal ganglia, including human autopsy studies. J Neurosurg 1967; 26: S112-126.

37. Mullan, S.: Percutaneous microcompression of the trigeminal ganglion. En Rovit RL, Murali R, Jannetta PJ (eds). Trigeminal neuralgia. Baltimore; Williams and Wilkins, 1990; pp. 137-144.

38. Mullan, S., Lichtor, T.: Percutaneous microcompression of the trigeminal ganglion for trigeminal neuralgia. $\mathrm{J}$ Neurosurg 1983; 59: 1007-1012.

39. Rovit, R.L.: Percutaneous radiofrequency thermal coagulation of the gasserian ganglion. En Rovit RL, Murali R, Jannetta PJ (eds). Trigeminal neuralgia. Baltimore; Williams and Wilkins, 1990; pp. 109-136.

40. Schmidek, H.H., Sweet, W.H. (eds): Operative neurosurgical techniques, 20 $0^{\text {th }}$ ed. Philadelphia; WB Saunders, 1988.

41. Shelden, C.H., Pudenz, R.H., Freshwater, D.B., Crue, B.L.: Compression rather than decompression for trigeminal neuralgia. J Neurosurg 1955; 12: 123-126.

42. Sweet, W.H.: The history of the development of treatment for trigeminal neuralgia. Clin Neurosurg 1985; 32: 294318 .

43. Sweet, W.H.: The treatment of trigeminal neuralgia (tic douloureux). N Engl J Med 1986; 315: 174-177.

44. Sweet, W.H., Wepsic, J.G.: Controlled thermocoagulation of trigeminal ganglion and rootlets for differential destruction of pain fibers. 1. Trigeminal neuralgia. J Neurosurg 1974; 40: 143-156.

45. Tobler, W.D., Tew, J.M. Jr, Cosman, E., Keller, J.T., Qualien, B.: Improved outcome in the treatment of trigeminal neuralgia by percutaneous stereotactic rhizotomy with a new, curved tip electrode. Neurosurgery 1983; 12: 313-317.

46. van Loveren, H., Tew, J.M. Jr, Keller, J.T., Nurre, M.A.: A 10-year experience in the treatment of trigeminal neuralgia. Comparison of percutaneous stereotaxic rhizotomy and posterior fossa exploration. J Neurosurg 1982; 57: 757-764.

47. Young, R.F.: The trigeminal nerve and its central pathways. Physiology of facial sensation and pain. En Rovit RL, Murali R, Jannetta PJ (eds). Trigeminal neuralgia. Baltimore; Williams and Wilkins, 1990; pp. 27-51.

48. Zakrzewska, J.M., Linskey, M.E.: Trigeminal neuralgia. Clin Evid (Online) 2009; pii: 1207.

Boto, G.R.: Neuralgia del trigémino. Neurocirugía 2010; 21: $361-372$.

Correspondencia: Gregorio Rodríguez Boto. Avda. Dr. García Tapia 159 D, $4^{\circ}$ B. 28030 Madrid.

E-mail: grboto@yahoo.es 